\title{
Computer-aided design system for GTE blades electrochemical machining technology
}

\author{
Maksim Nekhoroshev ${ }^{1, *}$, Nikolay Pronichev ${ }^{1}$, and Gennadiy Smirnov ${ }^{1}$ \\ ${ }^{1}$ Samara University, 34 Moskovskoye Shosse, Samara, 443086, Russia
}

\begin{abstract}
The paper presents the results of computer-aided design of GTE blade electrochemical machining. Developed a method to use ANSYS to calculate electric fields in the electrochemical cello under ECM. We created an electronic reference base and refined the dissolution model applicably to PECM. In addition, we developed a method for profiling tool electrodes DC ECM and PECM. The calculation module uses parametric relationships with Siemens NX by means of Excel files to produce output TE designs as a 3D computer models. The subsystem developed contains an ECM shaping model, has a modular structure, and is implemented as a wizard.
\end{abstract}

\section{Introduction}

When creating a generic methodology for computeraided design of ECS operations for geometrically complex parts, where computational technology is applied end-to-end, one needs to address a set of design problems, e.g. to design a methodology for calculating the profile of a tool electrode using CAE systems.

Papers $[1,2]$ give a detailed description of methods for computer-aided design of DC ECS operations. As current industries widely employ impulse ECS for geometrically complex parts being made of hard-tomachine materials, the process necessitates integrated research for computer-aided design of such operations with the use of CAD/CAM/CAE/PLM systems. PECSmachined surfaces dissolve in a non-stationary environment. All electrode processes occur within the flow of current and re-occur periodically. During pauses, surfaces stop dissolving while the gradients of ion concentrations in the near-electrode layers decrease; polarization-caused potential steps alter. When modeling the process of electrochemical dissolution, theoretical description of its kinetics is impossible. For practical use, we developed a theoretical-experimental model for calculating PECS shaping; the model should enable sufficiently accurate virtual profiling of tool electrodes (TE).

When developing the model, we assumed the following:

- electrodes were profiled at specified surface points, the coordinates of which were changed sequentially;

- the entire machining process was broken down into short intervals equal to the duration of the pulse package (for better accuracy, cycles can be as short as a single pulse);

- to calculate the removal per cycle for each point, the process flow conditions were assumed to be quasi- stationary, while the interelectrode gap (IEG) was assumed to be constant;

- for the assumed duration of machining cycles, for each point the potential steps were determined from the experiment as the averaged value $\varphi_{\text {eqv }}$.

- a standard experiment to determine $\varphi_{\text {eqv }}$ was carried out for each machine whereby power supply pulse parameters as well as experiment conditions were known.

- the dependency $j=f(a)$ was read, where

$j$ is the current density, $a$ is the interelectrode gap;

- all ECS parameters were consistent with the previously accepted values and remained constant; the gap changed discreetly within the specified limits;

- time to record each current value was minimized to ensure the condition $a=$ const would hold for each point.

\section{Materials and methods}

When developing methods for automated design of 3D EC shaping operations, we carried out an informational and analytical review of approaches to similar problems.

In paper [3], the authors propose a model that was developed when solving the related problems above, whereby some parameters were either assumed to be constant, or the range within which they could change would be pre-specified based on other parameters and preliminary experimental research of samples, machined correspondingly. The authors modeled the shaping process for the specified tool electrode profile as well as copying the TE profile using a reference blade.

Paper [4] is also about solving the problem of TE profiling based on modeling the processing occurring in IEG. For better accuracy of the model, the surface machined was discretized to improve the accuracy of computer modeling. They have also developed a special shell to simplify the construction of free surfaces.

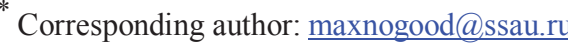


Paper [5] dwells upon special physical and mathematical models used to develop a computer-aided ECS process design system module. The module enables the simulation of processes occurring in an electrochemical IEG.

Paper [6] covers the issue of optimizing the electrochemical machining of hard-to-machine materials. Its authors have developed a mathematical algorithm and obtained mode parameters regression equations. They believe that using this algorithm reduces the time to find the optimal parameters, which is crucial for cost reductions in ECS operations design.

In paper [7], they used 3D ECS modeling with volumetric distribution of parameters in IEG being factored in; however, the calculation process itself would become significantly longer.

In papers on similar topics $[8,9,10,11]$, they solved the problems of modeling the ECS process in geometrically complex IEG, an inter-blade gap of a mono-wheel, to describe the complex electrode trajectory. Simulation-obtained data were used in $\mathrm{CAD} / \mathrm{CAM}$ systems for $\mathrm{CNC}$ machining. In the process of modeling, some parameters were assumed to be constant and would be determined by preliminary experiments. The solution optimized the electrolyte flow rate as well as the shape of the tool electrode.

In papers $[12,13]$, the researchers determined the optimal blade airfoil machining parameters to minimize the labor intensity and maximize the cost-effectiveness of the process. This principle can be used to optimize the ECS process.

In particular, results of researching titan alloys $[14,15]$ were used to define the parameters of machined materials.

Summarizing the above, it is safe to say that ECS modeling, TE profiling, and optimization of machining parameters are topical problems that many authors are striving to solve while using different approaches.

The method we propose herein is based on mathematical models as well as analyzing the spatial evolution of arbitrary surfaces and the physico-chemical processes in IEG and near-electrode layers.

\section{Creating a method for computer-aided ECS of GTE blades}

In accordance with the standard experiment methodology designed, we carried out research of PECS conditions for $\mathrm{NiCr}$ alloys. These materials are widely used for making GTE blades. Fig. 1 presents the results for EI-961 steel and EP-718 alloy. Experiment results were processed using the methods presented in $[1,2]$ and automatically recorded in the Teamcenter database, see Fig. 2.

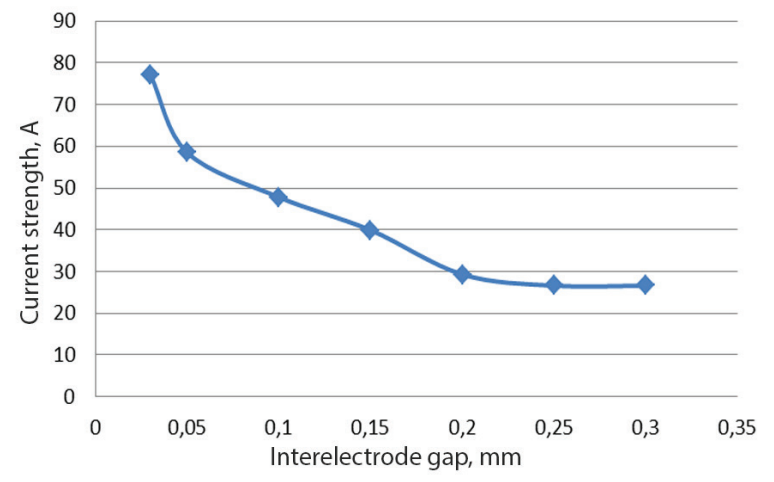

a

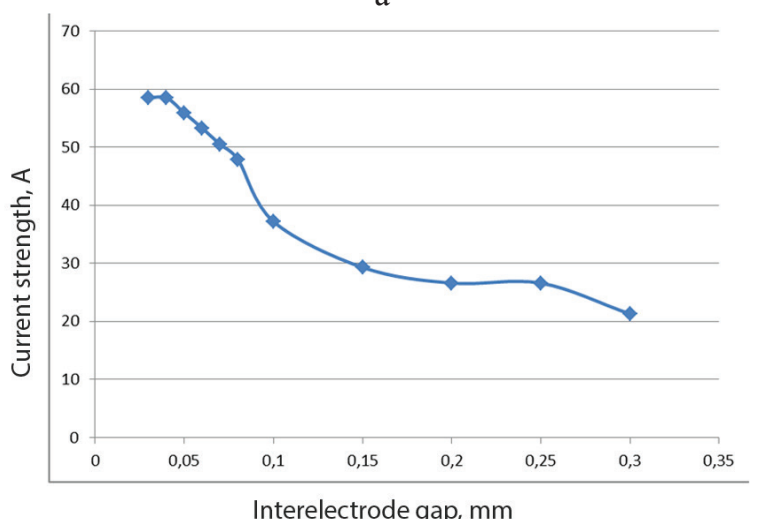

b

Fig. 1. Dependency of amplitude current on the inter-electrode gap for alloys: a. EI-961, b. EP-718.

To solve the problems of computer-aided design of GTE blade electro-chemical machining technology, we:

1. Developed a method to use ANSYS to calculate electric fields in the electrochemical cell under ECS. The calculations made confirmed the results obtained by analyzing resistance paper and showed that the computer model was consistent with the law of potential (current) distribution in the electrochemical cell. Based on this research, we created a database with material and electrolyte characteristics, dependencies of current output, and polarization curves for DC ECS. These data were presented in a tabular format for subsequent iterative calculations in ANSYS [1,2].

2. We also experimented to make readings of pulse current characteristics in the entire range of working gaps for various materials and electrolytes so as to create databases for computer-aided design. We also created an electronic reference base and refined the dissolution model applicably to PECS.

3. We developed a method for profiling tool electrodes for DC ECS and PECS. This methodology is based on calculating the TE profile when reverse-copied using the CAD model developed. It is essentially about iterative adjustments of TE shape. For ECS TE design, we chose a GTE blade airfoil as a sample $[1,2]$.

4. To facilitate the use of this method for factory technologists, we developed an application that simplified running the algorithm and reduced the modeling process to a wizard that had simplified 
standard settings. The module profiles tool electrodes by reverse copying for DC ECS and dimensional pulse ECS of GTE compressor blade airfoils.

5. The calculation module uses parametric relationships with Siemens NX by means of Excel files to produce the output TE designs as 3D computer models. For an example, we created a model of tool electrodes for GTE blades.

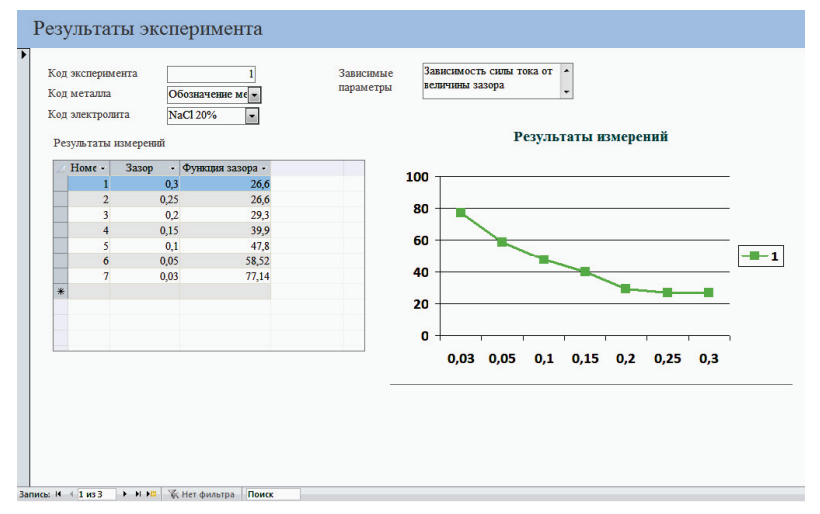

Fig. 2. Adding the experimental data to the database.

To improve the efficiency of using this output, we had to create a Siemens NX information parameters model for $\mathrm{CNC}$ shaping of tool electrodes designed based on computer modeling of GTE blade ECS. This model can be implemented in the environment of the calculation module developed earlier that is used to profile tool electrodes for pulse electrochemical machining of GTE blades, whereby the mathematical model developed is applied.

Let us analyze this computer-aided ECS design method as applicable to ECS of GTE blade airfoils, as it is such geometrically complex surfaces that reveal all problems that may arise in design. Below are the detailed steps of how the computer-aided design system was created.

Blade airfoil surfaces models and workpieces to machine were generated in the integrated CAE system. Current density was calculated iteratively in ANSYS with machining parameters being factored in. Based on the distribution of potentials and currents, the profile of the workpiece surfaces machined would change. Calculations were iterated until the first point of the dissolution boundary was on the lower blade airfoil tolerance limit.

The computer-aided ECS design subsystem solves both its primary problem (TE profiling) and the reverse problem of evaluating the geometric parameters of blade airfoils machined with TE of specified profile. When developing such a subsystem, it is important to implement wizard-like modeling, as the entire design process includes a set of interrelated procedures [1,2], which enables 'virtual' electrochemical machining of blades. Essentially, implementing a design wizard means that machining of test parts to refine the process is replaced with 'machining' their geometric models in calculational experiments.

Shaping analysis is crucial for process design because at the modeling stage it enables one to evaluate the possibility of making appropriate blades where allowance values are known, with the accuracy of blade geometry being factored in. This is an important issue for designing a blade ECS technology for low-allowance workpieces. Localization of machining depends on the properties of electrolyte, machined material, and machining parameters. When using ECS, the structure of minimum allowance changes as it has to include an additional component with the real localization characteristic being factored in. The properties of the Machined Material - Electrolyte system were determined experimentally and added to our Teamcenter database, where they are highlighted in the Experiment table.

Computer-aided TE profiling is the most complex and demanding problem. To solve it accurately means to face significant challenges due to the necessity to factor in the changes in dissolution rates at various points on the movable boundary. On this basis, for automating the design of EC shaping operations we used an approach where the reverse EC shaping problem was down to solving a chain of direct problems, i.e. we used the method of sequential iterations. Based on their solutions, we adjusted the working surface of TE as well as the machining parameters.

The output of the computer-aided EC shaping design subsystem is geometric models of the workpiece and the profile part of tool electrodes, machining parameters, and other technical data. These data were transmitted to the integrated $\mathrm{CAD} / \mathrm{CAM}$ system to design a TE-making technology, in particular to develop CNC programs to obtain the shaping surfaces of TE.

When creating the computer-aided ECS design subsystem, we developed information models of DC ECS and PECS shaping [1].

These two methods have a common basis; the distribution of potentials and currents in IEG was simulated using ANSYS. However, the assumptions we made and the boundary condition determination principles we adopted to solve this engineering problem were significantly different from what the authors of $[1,2]$ describe.

For the dissolution of surfaces of DC-machined workpieces, the dependency of potential on the current density was assumed as the boundary condition and presented in the form of polarization curves for the specified part material and electrolyte. These data were chosen automatically from the Teamcenter databases.

When analyzing EC shaping operations, processes with considerably different time and space scales would be calculated using different discretization intervals for time and space.

As the evolution (and thus the IEG configuration) of machined surfaces occurs at a considerably lower rate than the rate at which hydrodynamic and diffusion processes achieve steady state, we assumed that EC shaping processes within the IEG occur in a quasistationary mode. 


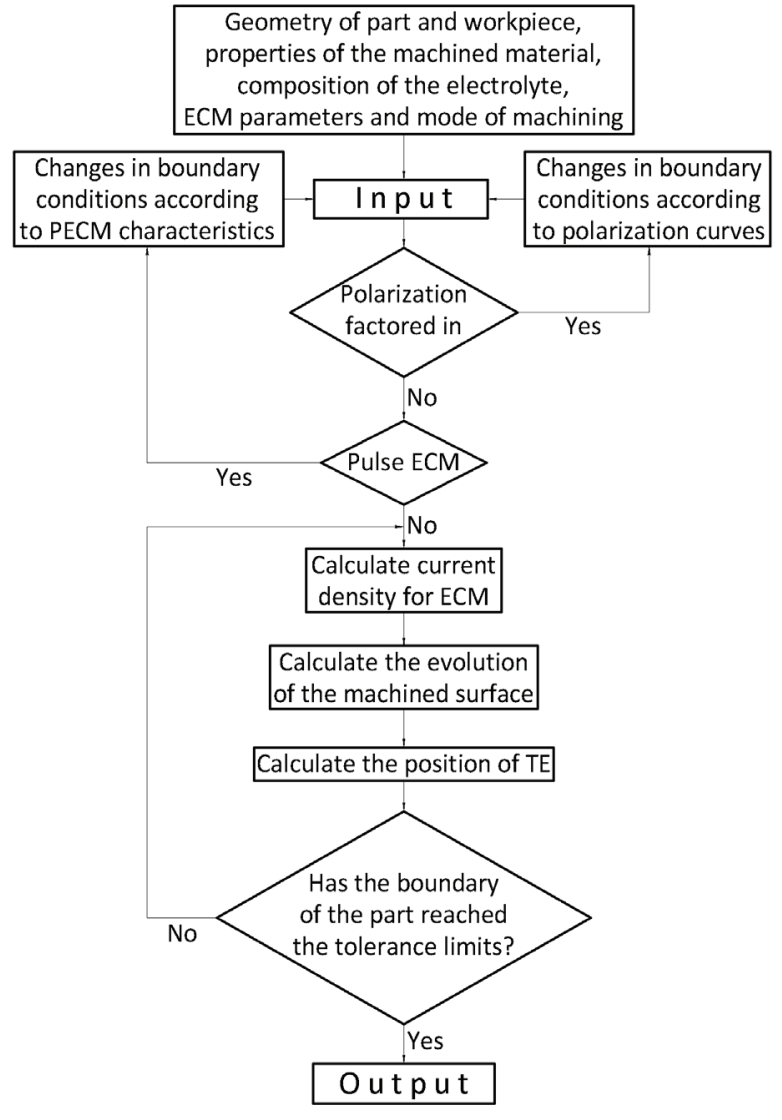

Fig. 3. EC shaping analysis chart

In line therewith, we implemented the following EC shaping analysis chart, see Fig. 3:

- calculate the current density in the IEG volume based on the ANSYS solution;

- interpret the values and directions of the current in each finite element mesh node into an etching boundary offset;

- determine the altered workpiece shape.

After the new position of the machined surface was determined, we checked whether the machining of the workpiece was complete. If machining was not complete, the calculation would be reiterated in the above sequence for the new position of the machined surface. Otherwise, calculations would stop and their results would be recorded in a file for further processing and visualization.

For pulse EC shaping, calculations are done similarly, but the anode and cathode potential values are picked from a database formed on the basis of experimental current readings.

To synthesize EC shaping operations, one has to determine the working surface of TE and the machining parameters, including the choice of EC shaping process (DC or pulse) to ensure that the specified surface would be produced with appropriate accuracy and maximized performance. Fig. 4 presents an EC shaping synthesis (design) chart.

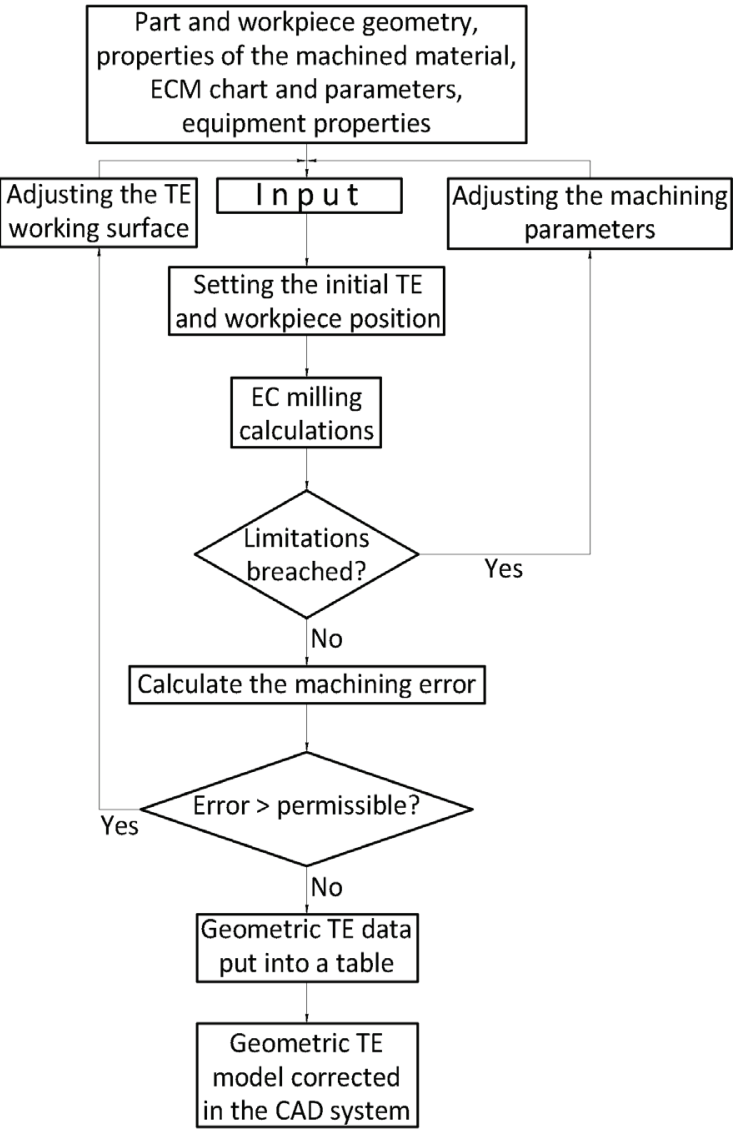

Fig. 4. EC shaping design chart

\section{Conclusion}

Therefore, this research summarizes all specific methods earlier developed and enables computer-aided design of GTE blade electrochemical machining, whether DC or pulse.

For more accurate profiling, we obtained experimental dependencies of current on the gap value for PECS operations performed with a pilot machine. Based on these studies, we calculated the values of $\varphi_{\text {eqv }}$ which were then used in EC shaping process models as boundary conditions. This approach factors in the mutual impact of parameters on the evolution of PECSdissolved surfaces.

\section{References}

1. M.V. Nekhoroshev, N.D. Pronichev and G.V. Smirnov, Open Mechan Engin. J. 8 (2014)

2. M.V. Nekhoroshev, N.D. Pronichev and G.V. Smirnov, Proceedings of the Samara Scientific Center, Russian Academy of Sciences, V. 15, 6(4) (2013)

3. F. Klocke, M. Zeis, S. Harst, A. Klink, D. Veselovac and M. Baumgärtner, Procedia CIRP, V. 8 (2013)

4. T. Paczkowskia and J. Zdrojewski, Procedia CIRP 42 (2016) 
5. J. Kozak, Lecture Notes in Electrical Engineering V. 170 (2013)

6. R. Mukherjee and Chakraborty, International J. of Advan. Manuf. Tech., V. 64, I. 5-8 (2013)

7. M. Purcar, A. Dorochenko, L. Bortels, J. Deconinck and B. Van den Bossche, J. of Mater. Proces. Techn., V. 203, I. 1-3 (2008)

8. J. Zhao, F. Wang, J. Xu and Y. Liu, Hangkong Xuebao/Acta Aeronautica et Astronautica Sinica, V. 34, I. 12 (2013)

9. F. Wang, J. Xu and J. Zhao, Hangkong Xuebao/Acta Aeronautica et Astronautica Sinica, V. 31, I. 12 (2010)

10. D. Zhu, D. Zhu and Z. Xu, Inter. J. of Adv. Manuf. Tech., V. 62, I. 1-4 (2012)

11. R.Wu, D. Zhang and J. Sun, Res. J. of Applied Sciences, Engin. and Tech., V. 3, I. 9 (2011)

12. E.M. Korovin, A.N. Lunev and V.V. Tsareva, Russian Aeronautics, V. 55, I. 1 (2012)

13. A.N. Lunev, L.T. Moiseeva, and M.V. Solomina, Russian Aeronautics, V. 50, I. 2 (2007)

14. A.I. Khaimovich and A.V.Balaykin, ARPN $J$ of Engin and Applied scien., №9(10) (2014)

15. A.V. Balaykin, E.A. Nosova and N.V. Galkina, $I O P$ Conf. Series: Mater. Sc. and Engin., V. 156, I 1 (2016) 